\title{
A technique for improved stability of adaptive feedforward controllers without detailed uncertainty measurements
}

\author{
A. P. Berkhoff \\ TNO Technical Sciences, Delft, Netherlands, email: arthur.berkhoff@tno.nl, and \\ University of Twente, Enschede, Netherlands, email: a.p.berkhoff@utwente.nl
}

\begin{abstract}
Model errors in adaptive controllers for reduction of broadband noise and vibrations may lead to unstable systems or increased error signals. Previous work has shown that the addition of a low-authority controller that increases damping in the system may lead to improved performance of an adaptive, high-authority controller. Other researchers have suggested to use frequency dependent regularization based on measured uncertainties. In this paper an alternative method is presented that avoids the disadvantages of these methods namely the additional complex hardware, and the need to obtain detailed information of the uncertainties. An analysis is made of an active noise control system in which a difference exists between the secondary path and the model as used in the controller. The real parts of the eigenvalues that determine the stability of the system are expressed in terms of the amount of uncertainty and the singular values of the secondary path. Based on these expressions, modifications of the feedforward control scheme are suggested that aim to improved performance without requiring detailed uncertainty measurements. For an active noise control system in a room it is shown that the technique leads to improved performance in terms of robustness and the amount of reduction of the error signals.
\end{abstract}

\section{Introduction}

Improved stability is desirable in many implementations of adaptive control algorithms based on the filtered-reference LMS algorithm or the filtered-error LMS algorithm. Preferably, such robustness improvements do not lead to increases of the error signal. In this document, some techniques for improved robustness are presented. For some control schemes, online adaptation of the model is possible in principle but a large amount of additional noise has to be injected in the system for rapid changes in the model [1]. Furthermore, if the controller uses model-based preconditioning or factorization, then these time-consuming operations should be performed online as well. Robust control approaches are described in, for example Ref. [2]. Probabilistic methods leading to frequency dependent regularization for optimum filtering are described by $[3,4]$. Methods for adaptive control are given in $[5,6]$. Such algorithms can be tuned for a particular application but require additional effort in the design stage and presume that sufficient a-priori knowledge is available about the uncertainty. An alternative approach is to use a high-authority and low-authority control (HAC/LAC) architecture [7,8] where the goal of the low-authority controller is to add active damping to the structure. Active damping can be implemented using different strategies. The use of a HAC/LAC architecture yields three major advantages [7]. Firstly, the active damping extends outside the bandwidth of the HAC control loop, which reduces the settling times outside the control bandwidth. Secondly, it is easier to gain-stabilize the modes outside the bandwidth of the outer loop. And thirdly, the large damping of the modes inside the controller bandwidth makes them more robust to parametric uncertainty. In this paper an example is given of an application in which an alternative approach is possible which does not require the complex hardware of a HAC/LAC scheme nor does it need detailed a-priori knowledge of the uncertainty.

\section{Methods}

\subsection{Adaptive feedforward controller}

The methods are tested in combination with a particular version of a filtered-error type LMS algorithm [9]. A block diagram of the multiple-input multiple-output adaptive controller is shown in Fig. 1. A detailed description of this algorithm can be found in Refs. [10, 11]. For the description of the MIMO controller, we assume that there are $K$ reference signals, $L$ error sensors and $M$ actuators. The transfer function between the actuators and the error sensors is denoted by the $L \times M$-dimensional secondary path $G$. Denoting $n$ as the sample instant, the update rule for the controller coefficients is

$$
W_{i}(n+1)=W_{i}(n)-\alpha e^{\prime \prime}(n) x^{\prime T}(n-i),
$$

in which the $i$-th filter coefficients of the control filters are represented by the $M \times K$ matrix $W_{i}$, where $i=0 . . N_{W}-1$, i.e., $W(q)=\sum_{i=0}^{N_{W}-1} q^{-i} W_{i}$, with $q$ the unit delay operator. Furthermore, $e^{\prime \prime}(n)$ is the $M \times 1$ vector of auxiliary error signals, $x^{\prime}(n)$ is the $K \times 1$ vector of delayed reference signals, and $\alpha$ is the convergence coefficient. In the actual implementation, a normalized LMS update rule was used, combined with 'leakage' of the control coefficients [6]. An 
allpass factor $\bar{G}_{i}$ and minimum-phase factor $\bar{G}_{o}$ are obtained from an inner-outer factorization such that $\bar{G}=\bar{G}_{i} \bar{G}_{o}$. The adjoint $\bar{G}_{i}^{*}$ is combined with a delay $D$ of $N_{D}$ samples in order to ensure that $D \bar{G}_{i}^{*}$ is predominantly causal. The transfer function $G_{r p}$ subtracts the contribution of the actuators on the reference signals, as required for internal model control (IMC) [12]. Although the algorithm differs in some aspects from the standard filtered-reference LMS of filtered-error LMS algorithms, especially with respect to the speed of convergence, the stability properties are governed by the same underlying equations. Therefore, for the remainder of this paper, it is assumed that the analysis applies to general filtered-reference LMS or filterederror LMS algorithms. Nevertheless, it will be seen that the algorithm of Fig. 1 allows for the implementation of the techniques discussed in this document. In particular, the scheme facilitates the implementation of frequency dependent regularization techniques, which can be useful for robust control approaches.

\subsection{Uncertainty and eigenvalue conditions}

We assume that the uncertainty $\Delta G$ in the secondary path $G$ is such that

$$
\widehat{G}=G+\Delta G
$$

in which $\widehat{G}$ is the model of the secondary path $G$. The condition for stability of the LMS update rule is determined by the minimum real part of any eigenvalue of a matrix determined by the secondary path and the model of the secondary path [6]:

$$
\min \operatorname{Re} \lambda\left\{\widehat{G}^{H}\left(e^{\mathrm{j} \omega}\right) G\left(e^{\mathrm{j} \omega}\right)\right\}>0 .
$$

Alternatively,

$$
\min \operatorname{Re} \lambda\left\{\left(G\left(e^{\mathrm{j} \omega}\right)+\Delta G\left(e^{\mathrm{j} \omega}\right)\right)^{H} G\left(e^{\mathrm{j} \omega}\right)\right\}>0 .
$$

If the system has a single input and a single output then the matrix $\widehat{G}^{H} G$ is a scalar quantity and it can be shown that the stability condition is equivalent to the requirement that the absolute value of the phase difference between $\widehat{G}^{H}\left(e^{\mathrm{j} \omega}\right)$ and $G\left(e^{\mathrm{j} \omega}\right)$ is smaller than $90^{\circ}$ at all frequencies [6].

A stronger condition is given by the SPR condition of Ren and Kumar [13]:

$$
\min \lambda\left\{\frac{1}{2}\left[\widehat{G}^{H}\left(e^{\mathrm{j} \omega}\right) G\left(e^{\mathrm{j} \omega}\right)+G^{H}\left(e^{\mathrm{j} \omega}\right) \widehat{G}\left(e^{\mathrm{j} \omega}\right)\right]\right\}>0 .
$$

or

$$
\begin{aligned}
& \min \lambda\left\{\frac { 1 } { 2 } \left[\left(G\left(e^{\mathrm{j} \omega}\right)+\Delta G\left(e^{\mathrm{j} \omega}\right)\right)^{H} G\left(e^{\mathrm{j} \omega}\right)\right.\right. \\
& \left.\left.+G^{H}\left(e^{\mathrm{j} \omega}\right)\left(G\left(e^{\mathrm{j} \omega}\right)+\Delta G\left(e^{\mathrm{j} \omega}\right)\right)\right]\right\}>0 .
\end{aligned}
$$

In the latter two equations, all eigenvalues $\lambda$ are real since the underlying matrix is Hermitian.

\section{Minimum-real eigenvalue for bounded uncertainty}

Let $G=G\left(e^{\mathrm{j} \omega}\right), \Delta G=\Delta G\left(e^{\mathrm{j} \omega}\right)$ and $G=U \Sigma V^{H}$ the singular value decomposition of $G$. The matrix $\Sigma$ contains the positive singular values $\sigma_{1} \geqslant \sigma_{2} \ldots \geqslant$ $\sigma_{M}$. The norm of the uncertainty is bounded such that for each angular frequency $\omega$

$$
\left\|\Delta G\left(e^{\mathrm{j} \omega}\right)\right\| \leqslant \rho(\omega)
$$

\subsection{Stability condition}

First, we will study the influence of uncertainty on the eigenvalues of $\widehat{G}^{H} G$ in Eq. (4). It will be shown that for any singular value $\sigma_{i}$ the eigenvalues are contained in a circle with centre $\sigma_{i}^{2}$ and radius $\rho \sigma_{i}$. Previous work can be found in Refs. [6, 13, 14]. The derivation below gives a direct proof of the desired property and avoids implicit or approximate derivations. An eigenvector $q$ and corresponding eigenvalue $\lambda$ of Eq. (4) satisfy

$$
(G+\Delta G)^{H} G q=\lambda q
$$

Right multiplication with $q^{H}$ leads to

$$
\lambda I-G^{H} G=\Delta G^{H} G .
$$

Use of the singular value decomposition $G=U \Sigma V^{H}$ allows us to write

$$
\lambda I-G^{H} G=V\left(\lambda I-\Sigma^{H} \Sigma\right) V^{H}
$$

where we have used $\lambda I=\lambda V V^{H}=V \lambda I V^{H}$. Then, left multiplication with $V^{H}$ and right multiplication with $V$ shows that

$$
\lambda I-\Sigma^{H} \Sigma=V^{H} \Delta G^{H} U \Sigma
$$

The $i$ th element on the diagonal of the matrix on the left hand side is $\lambda-\sigma_{i}^{2}$. This element is obtained by selecting the $i$ th row $V(:, i)^{H}$ of the matrix $V^{H}$ and the $i$ th column $\Sigma(:, i)$ of the matrix $\Sigma$ :

$$
\lambda-\sigma_{i}^{2}=V(:, i)^{H} \Delta G^{H} U \Sigma(:, i)
$$

The norm satisfies

$$
\left|\lambda-\sigma_{i}^{2}\right| \leqslant\left\|V(:, i)^{H}\right\|\left\|\Delta G^{H} U \Sigma(:, i)\right\|
$$

which, because $\left\|V(:, i)^{H}\right\|=1$ and $\| \Delta G^{H} U \Sigma($ : $, i) \| \leqslant \rho \sigma_{i}$ can be written as

$$
\left|\lambda-\sigma_{i}^{2}\right| \leqslant \rho \sigma_{i}
$$


Hence, the minimum real part of $\lambda$ within the circle corresponding to singular value $\sigma_{i}$ is obtained for $\sigma_{i}^{2}-\rho \sigma_{i}$, and the overall minimum is obtained by evaluating all circles:

$$
\min \operatorname{Re} \lambda=\min _{i} \sigma_{i}\left(\sigma_{i}-\rho\right)
$$

If a singular value $\sigma_{i}$ exists for which $\rho>\sigma_{i}$ then the system is unstable.

\subsection{SPR condition}

Next, let us consider Eq. (6). An eigenvector $q$ and corresponding eigenvalue $\lambda$ of Eq. (6) satisfy

$$
\left.\frac{1}{2}\left[(G+\Delta G)^{H} G+G^{H}(G+\Delta G)\right)\right] q=\lambda q .
$$

Right multiplication with $q^{H}$ leads to

$$
r^{H} r+\frac{1}{2}\left(s^{h} r+r^{h} s\right)=\lambda
$$

in which we have used $r=G q$ and $s=\Delta G q$. Then,

$$
\min \lambda=\min \left\{r^{H} r+\frac{1}{2}\left(s^{H} r+r^{H} s\right)\right\}
$$

For the trivial case $r=0$, the minimum eigenvalue is therefore $\lambda=0$. Furthermore, the method of completing the squares shows that

$$
\min \lambda=\min \left\{\left(r+\frac{1}{2} s\right)^{H}\left(r+\frac{1}{2} s\right)-\frac{1}{4} s^{H} s\right\}
$$

Provided there are no constraints for $r$, the minimum eigenvalue is obtained for $r=-\frac{1}{2} s$. Because $\|s\| \leqslant$ $\|\Delta G\|\|q\| \leqslant \rho$, then also

$$
\min \lambda=-\frac{1}{4} \rho^{2}
$$

for $\|r\|=\rho / 2$. In order to find the minimum eigenvalue in case of constraints for $r$ we will try to find a relationship between $r$ and $s$ at the minimum. We assume

$$
s=\alpha r+\alpha_{\perp} r_{\perp}
$$

in which $r$ and $r_{\perp}$ are nonvanishing and in which $r$ and $r_{\perp}$ are orthogonal, i.e. $r_{\perp}^{H} r=0$. We try to find the minimizing values for $\alpha$ and $\alpha_{\perp}$. Substitution of Eq. (21) in Eq. (18) shows that

$$
\begin{aligned}
\min \lambda=\min \left\{r^{H} r\right. & +\frac{1}{2}\left(\left(\alpha r+\alpha_{\perp} r_{\perp}\right)^{H} r\right. \\
& \left.\left.+r^{H}\left(\alpha r+\alpha_{\perp} r_{\perp}\right)\right)\right\}
\end{aligned}
$$

Hence,

$$
\min \lambda=\min \|r\|^{2}(1+\alpha)
$$

which has a minimum value for minimum $\alpha$, which is also real since $\lambda$ is real. Furthermore,

$$
\|s\|^{2}=\|\alpha\|^{2}\|r\|^{2}+\left\|\alpha_{\perp}\right\|^{2}\left\|r_{\perp}\right\|^{2}
$$

Therefore, since $\|s\|^{2} \leqslant \rho^{2}$

$$
\|\alpha\|^{2}\|r\|^{2} \leqslant \rho^{2}-\left\|\alpha_{\perp}\right\|^{2}\left\|r_{\perp}\right\|^{2}
$$

Hence, the maximum $\|\alpha\|^{2}$ which is required for minimum $\alpha$ is obtained if

$$
\alpha_{\perp}=0
$$

Therefore

$$
\min \alpha=-\frac{\rho}{\|r\|}
$$

Hence, the optimum for $s$ is given by

$$
s=-\rho \frac{r}{\|r\|}
$$

and the minimum eigenvalue is

$$
\min \lambda=\|r\|(\|r\|-\rho)
$$

It can be verified that the unconstrained minimum $-\rho^{2} / 4$ is obtained for $\|r\|=\rho / 2$. If $\|r\|$ is constrained then the minimum eigenvalue is a function of $\|r\|$, which in turn depends on the singular values of $G$ since $\|r\|=\|G q\| \leqslant\|G\|$. Therefore, the norm of $r$ is in the range $\sigma_{M} \leqslant\|r\| \leqslant \sigma_{1}$. For small uncertainty when the smallest singular value $\sigma_{M}$ is at least $\rho / 2$ then the minimum eigenvalue follows from $\left.\|r\|(\|r\|-\rho)\right|_{\|r\|=\sigma_{M}}$ i.e.

$$
\min \lambda=\sigma_{M}\left(\sigma_{M}-\rho\right), \quad \rho \leqslant 2 \sigma_{M}
$$

For large uncertainty when the largest singular value $\sigma_{1}$ is smaller than or equal to $\rho / 2$ then the minimum eigenvalue follows from $\left.\|r\|(\|r\|-\rho)\right|_{\|r\|=\sigma_{1}}$. i.e.

$$
\min \lambda=\sigma_{1}\left(\sigma_{1}-\rho\right), \quad \rho \geqslant 2 \sigma_{1}
$$

If a singular value $\sigma_{i}$ exists for which the SPR condition fails, i.e., for which $\min \lambda<0$, then convergence of the adaptive algorithm may show overhoot but the system may still be stable [14].

\section{Controller modifications}

\subsection{Regularization}

Regularization can be used to ensure that all real parts of the eigenvalues are positive. The regularization can be implemented by defining an augmented plant $\bar{G}(q)$ :

$$
\bar{G}(q)=\left[\begin{array}{c}
G(q) \\
G_{\mathrm{reg}}(q)
\end{array}\right],
$$


in which the $L \times M$-dimensional secondary path $G(q)$ is augmented with an $L^{\prime} \times M$-dimensional transfer function $G_{\text {reg }}(q)$. This augmented plant allows us to define a cost function

$$
J=\mathrm{E}\left(\bar{e}^{T} \bar{e}\right)
$$

in which

$$
\bar{e}=\left[\begin{array}{c}
e \\
e_{\mathrm{reg}}
\end{array}\right]
$$

Hence

$$
J=\mathrm{E}\left(e^{T} e\right)+\mathrm{E}\left(e_{\text {reg }}^{T} e_{\text {reg }}\right)
$$

The error signal $e$ is defined as usual

$$
e=G u+d
$$

whereas the regularizing error signal is obtained from

$$
e_{\text {reg }}=G_{\text {reg }} u
$$

The requirement for stability now becomes

$$
\min \operatorname{Re} \lambda\left\{\widehat{\bar{G}}^{H}\left(e^{\mathrm{j} \omega}\right) \bar{G}\left(e^{\mathrm{j} \omega}\right)\right\}>0 .
$$

in which

$$
\widehat{\bar{G}}(q)=\left[\begin{array}{c}
\widehat{G}(q) \\
G_{\mathrm{reg}}(q)
\end{array}\right] .
$$

The stability condition can be written as

$\min \operatorname{Re} \lambda\left\{\widehat{G}^{H}\left(e^{\mathrm{j} \omega}\right) G\left(e^{\mathrm{j} \omega}\right)+G_{\mathrm{reg}}^{H}\left(e^{\mathrm{j} \omega}\right) G_{\mathrm{reg}}\left(e^{\mathrm{j} \omega}\right)\right\}>0$.

The SPR condition becomes

$\min \lambda\left\{\frac{1}{2}\left[\widehat{\bar{G}}^{H}\left(e^{\mathrm{j} \omega}\right) \bar{G}\left(e^{\mathrm{j} \omega}\right)+\bar{G}^{H}\left(e^{\mathrm{j} \omega}\right) \widehat{\bar{G}}\left(e^{\mathrm{j} \omega}\right)\right]\right\}>0$,

which can be written as

$$
\begin{array}{r}
\min \lambda\left\{\frac{1}{2}\left[\widehat{G}^{H}\left(e^{\mathrm{j} \omega}\right) G\left(e^{\mathrm{j} \omega}\right)+G^{H}\left(e^{\mathrm{j} \omega}\right) \widehat{G}\left(e^{\mathrm{j} \omega}\right)\right]+\right. \\
\left.G_{\text {reg }}^{H}\left(e^{\mathrm{j} \omega}\right) G_{\mathrm{reg}}\left(e^{\mathrm{j} \omega}\right)\right\}>0 .
\end{array}
$$

As compared to Eqs. (3) and (5) the eigenvalues of Eqs. (40) and (42), respectively, are modified by the $M \times M$-dimensional matrix $G_{\text {reg }}^{H}\left(e^{\mathrm{j} \omega}\right) G_{\text {reg }}\left(e^{\mathrm{j} \omega}\right)$. The task is to determine a minimum matrix $\Lambda=G_{\text {reg }}^{H} G_{\text {reg }}$ for each $\omega$ such that the selected condition holds. A diagonal matrix for $\Lambda$ should be sufficient, but the elements on the diagonal are not necessarily identical.

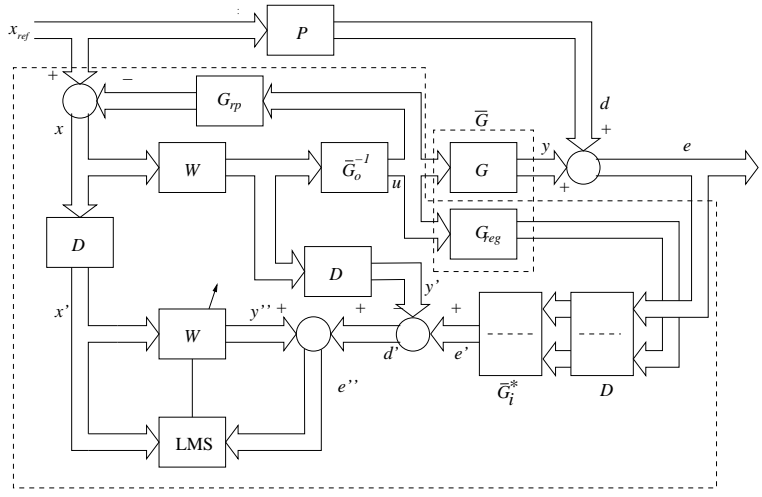

Figure 1: Regularized modified filtered-error adaptive control scheme with IMC [10, 11].

Spectral factorization of $\Lambda$ then leads to $G_{\mathrm{reg}}$. In practical situations, considerable time and effort is required to obtain sufficient information about the different conditions such that a reliable estimate of $\Lambda$ can be obtained. If $\Lambda$ is to be determined from the transfer function deviations for all possible conditions that may occur during operation, then this approach may be too time-consuming for many applications since each individual installation may require such an a-priori procedure.

\subsection{Damping by state feedback}

In resonant systems, improved robustness of adaptive algorithms can be achieved by increasing the damping of $G$ [15], which can be realized with separate highspeed control loops in a so-called HAC/LAC strategy [16]. For an adaptive feedback controller applied to a panel with piezoelectric actuators it was found [15] that increased robustness could be obtained if damping was active in the identification phase only, i.e., active damping was not applied during adaptive control. This implies that improved robustness could also be obtained if damping is applied numerically to the transfer function $G$. The full HAC-LAC control strategy still results in better performance and robustness properties [15]. Nevertheless, addition of damping to the transfer functions may lead to useful improvement of robustness, as demonstrated in the application of Ref. [15] . In this section we try to realize such a numerical damping to the model, in this case for an adaptive feedforward controller.

Let $x$ be the state vector and $u$ the actuator driving signals. Let $A, B, C, D$ be the state space system describing the system $G$. Feedback control can be implemented by using an LQR regulator [2] which determines the feedback gain $K$ defined by

$$
u=-K x
$$


minimizing the cost function

$$
J=\sum_{n}\left[x^{T}(n) Q x(n)+u^{T}(n) R u(n)\right]
$$

subject to

$$
x(n+1)=A x(n)+B u(n)
$$

The approach to realize damping is to set the feedback gain to a relatively low value, otherwise additional resonances with low damping may be introduced. A relatively low feedback gain $K$ is obtained by setting the weighting by $R$ relatively high as compared to the weighting by $Q$. The new state-space system with such feedback is obtained by setting:

$$
\begin{aligned}
& A \leftarrow A-B K \\
& B \leftarrow B \\
& C \leftarrow C-D K \\
& D \leftarrow D
\end{aligned}
$$

Alternatively, one could use feedback of the output $y$ such that

$$
J=\sum_{n}\left[y^{T}(n) Q^{\prime} y(n)+u^{T}(n) R^{\prime} u(n)\right]
$$

in which $y=C x+D u$. If $G$ contains significant phase delays then the $\mathrm{LQR}$ regulator could be applied to the minimum-phase factor $G_{o}$ only.

\section{System design based on measured transfer functions}

This section presents a stability analysis for an active noise control system in a room. The active noise control system uses 3 loudspeakers and 4 sensors. The sensor signals are a pressure signal and 3 particle velocity signals. The 4 sensors are positioned very close to each other using a Microflown USP probe. Experiments were performed to obtain transfer functions under different conditions. The secondary path was estimated using subspace identification techniques [17] based on Slicot (www.slicot.org). The accuracy of the transfer functions for white noise input was such that the Variance Accounted For (VAF) was approximately $99 \%$. The nominal situation consists of a room in which the door and the window are closed. The dimensions of the room are $5 \mathrm{~m} \times 3 \mathrm{~m} \times 2.6 \mathrm{~m}$. The loudspeakers are located in corners on the floor of the room, while the sensor is located near the longest wall at about $1 \mathrm{~m}$ from the wall and at a height of $1 \mathrm{~m}$. An example of a transfer function, for actuator 1 and sensor 1, is given in Figs. 2 and 3. The difference of the phase as compared to nominal condition is shown in Fig. 4.

The minimum real part of the eigenvalue according to Eqs. (3) and (5) for different conditions is given

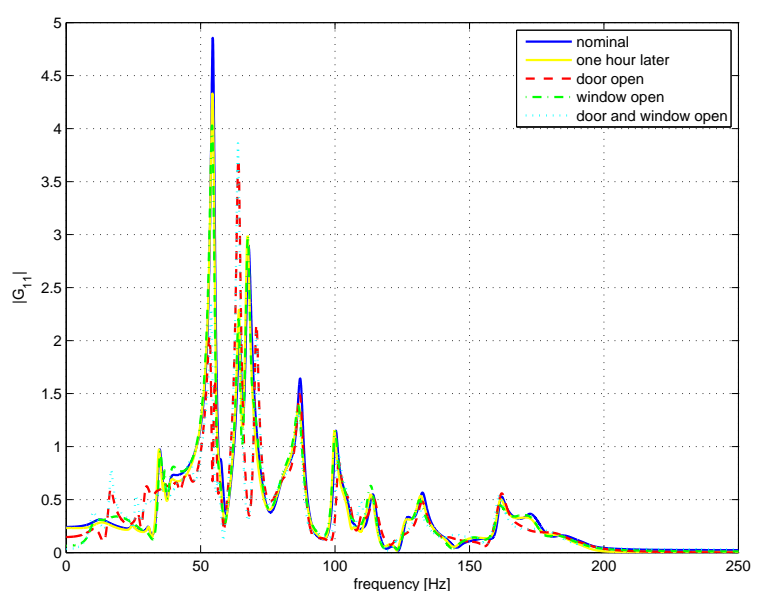

Figure 2: Magnitude of the transfer function between actuator 1 and sensor 1 for different conditions.

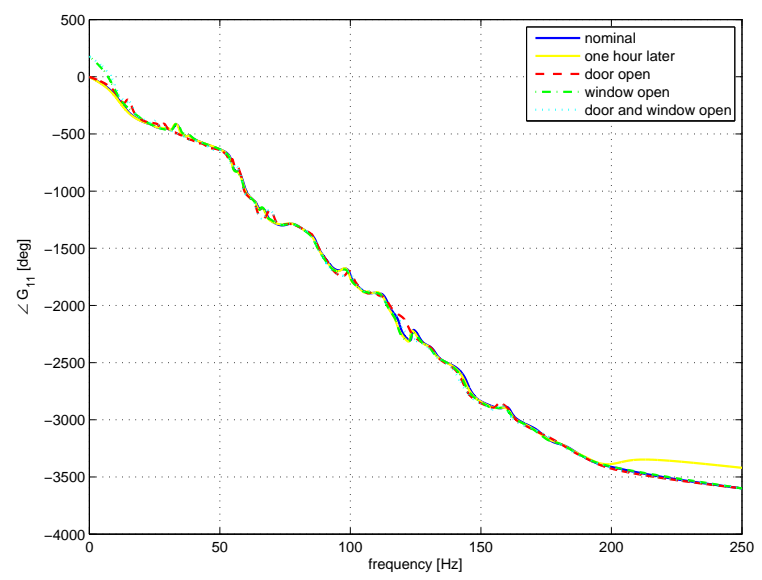

Figure 3: Phase of the transfer function between actuator 1 and sensor 1 for different conditions.

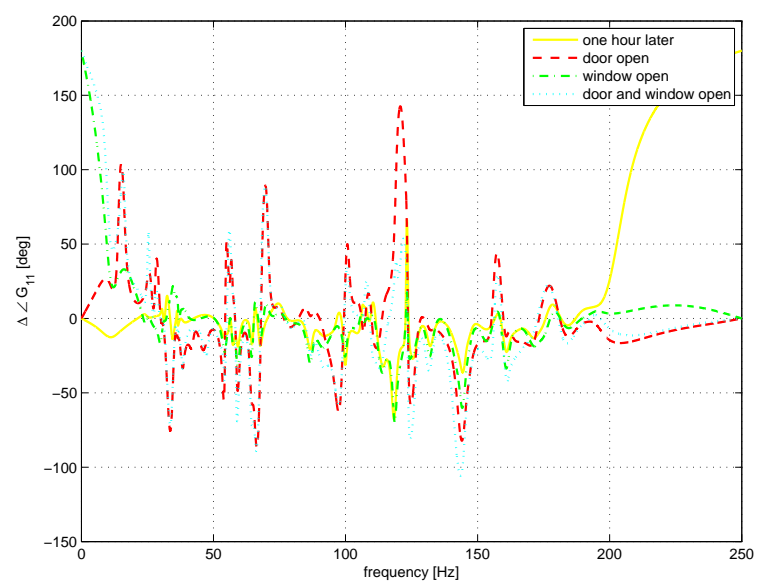

Figure 4: Difference of the phase of the transfer function between actuator 1 and sensor 1 for different conditions as compared to the nominal condition. 


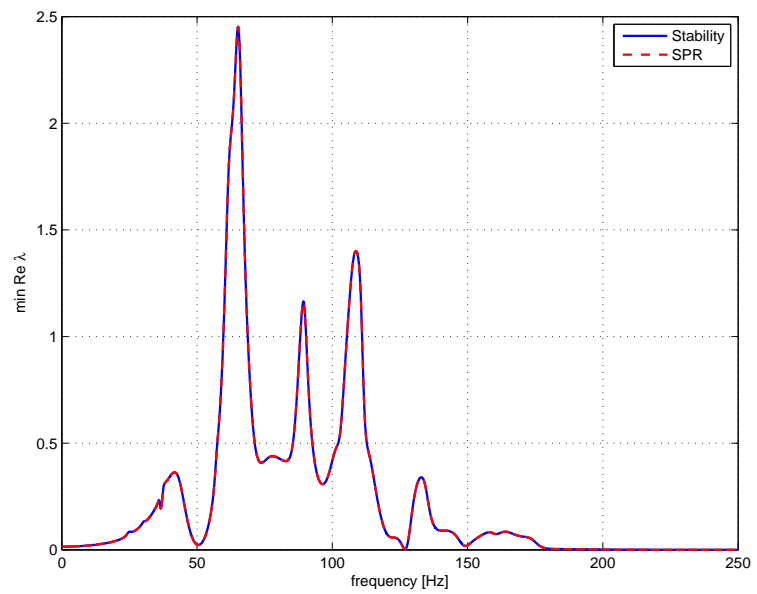

Figure 5: Minimum real eigenvalue of Eqs. (3) and (5) for a secondary path $G$ obtained in a room for the nominal situation and without model mismatch, i.e. $\widehat{G}=G$.

in Figs. 5 - 9. Fig. 5 shows the situation for which $\widehat{G}=G$. As a result all real parts of the eigenvalues are positive and the system is stable. Fig. 6 shows the situation for which the transfer functions are measured at two different instants with one hour in between but for which the conditions are the same. It can be seen that both for the stability condition and the SPR condition the minimum real part of the eigenvalues are positive for all frequencies, i.e., the system is expected to be stable. Fig. 7 shows the situation for the case that a door is fully opened. It can be seen that the abscissa is negative for some frequencies, leading to possibly unstable behavior. Problematic frequencies according to the stability condition are $36 \mathrm{~Hz}$ and $69 \mathrm{~Hz}$. Problematic frequencies according to the SPR condition are $37 \mathrm{~Hz}, 54 \mathrm{~Hz}, 69 \mathrm{~Hz}, 109 \mathrm{~Hz}$, and $124 \mathrm{~Hz}$. Fig. 8 shows the results for the minimum real eigenvalue for the case that a window is opened. In this case stability problems are expected at very low frequencies from $0 \mathrm{~Hz}$ to $5 \mathrm{~Hz}$ according to the stability condition and from $0 \mathrm{~Hz}$ to $7 \mathrm{~Hz}$ according to the SPR condition. Fig. 9 shows the minimum real eigenvalue for the case that both the door and the window are open. According to the stability condition, problematic frequencies are the range of $0 \mathrm{~Hz}$ to $9 \mathrm{~Hz}, 36 \mathrm{~Hz}$ and $69 \mathrm{~Hz}$. According to the SPR condition, problematic frequencies are the range of $0 \mathrm{~Hz}$ to $9 \mathrm{~Hz}, 16 \mathrm{~Hz}, 36 \mathrm{~Hz}, 40 \mathrm{~Hz}$, the range of $54 \mathrm{~Hz}$ to $56 \mathrm{~Hz}, 69 \mathrm{~Hz}, 108 \mathrm{~Hz}, 125 \mathrm{~Hz}$ and $147 \mathrm{~Hz}$.

Figs. 10 and 11 show the magnitude of the transfer function and the impulse response, respectively, for the secondary path without damping and the secondary path with damping. It can be seen that damping particularly reduces the peaks of the frequency domain response. It can also be seen that the impulse responses become shorter when damping is added.

The minimum real part of the eigenvalue according to Eqs. (3) and (5) for different conditions using

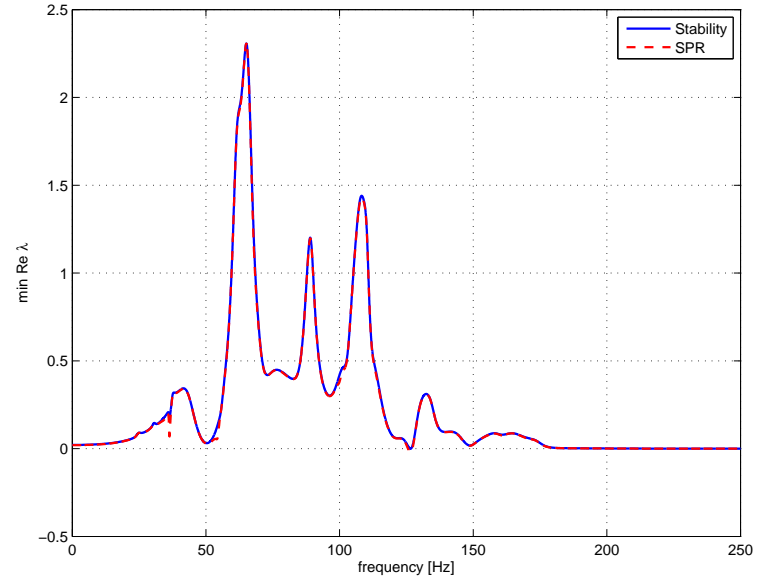

Figure 6: As Fig. 5, except that $G$ is obtained one hour later.

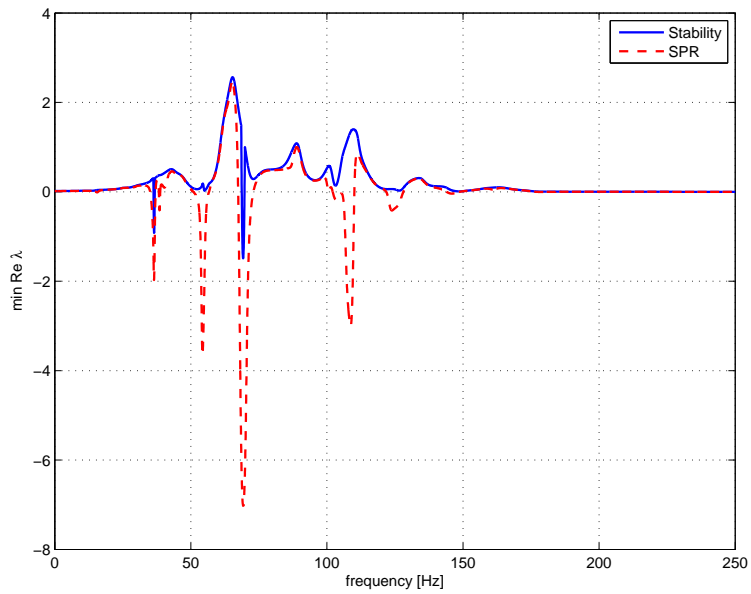

Figure 7: As Fig. 5, except that $G$ is obtained with the door open.

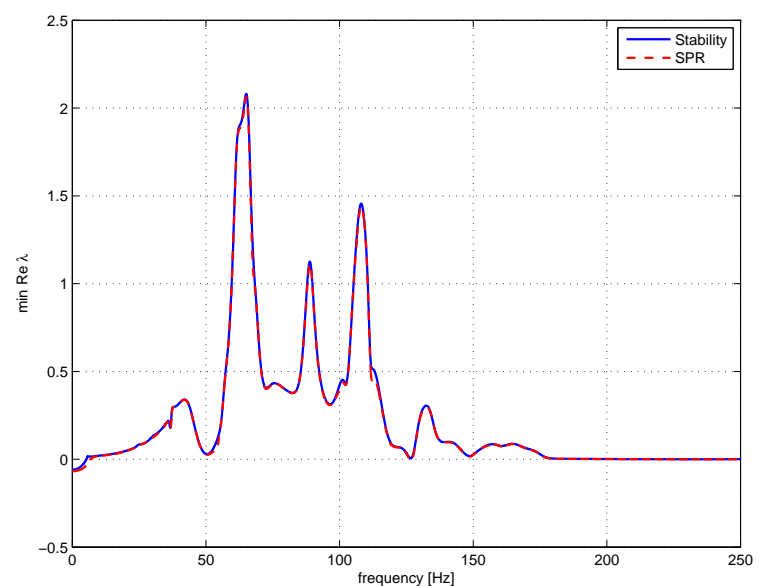

Figure 8: As Fig. 5, except that $G$ is obtained with the window open. 


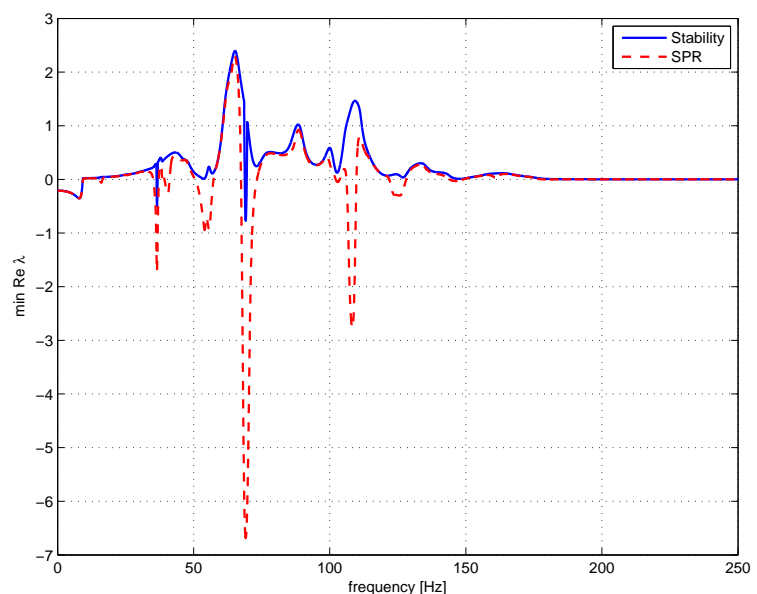

Figure 9: As Fig. 5, except that $G$ is obtained with the door and the window open.

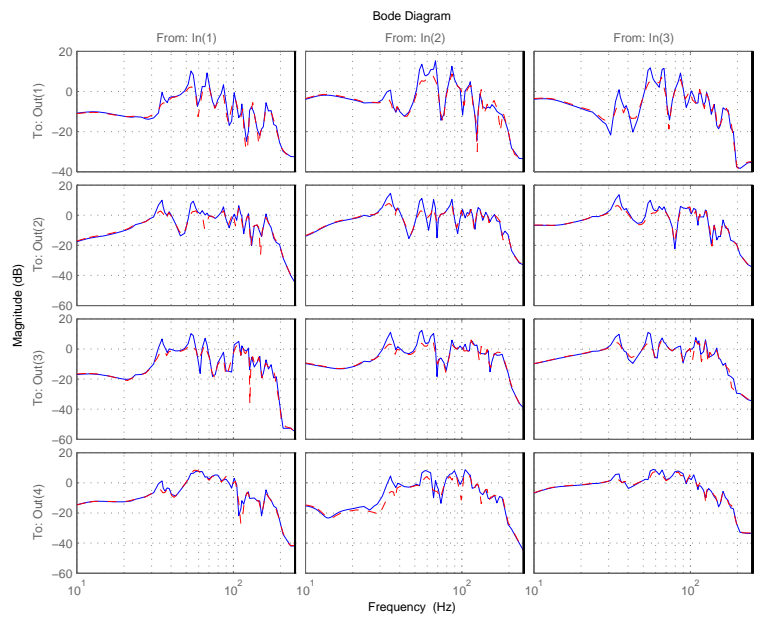

Figure 10: Magnitude of $G$ without damping (solid line) and with damping (dashed line).

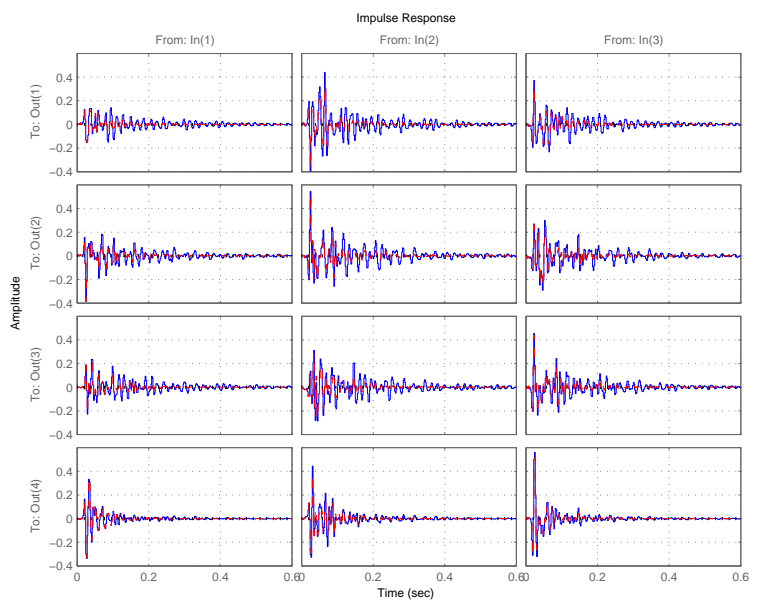

Figure 11: Impulse response of $G$ without damping (solid line) and with damping (dashed line).

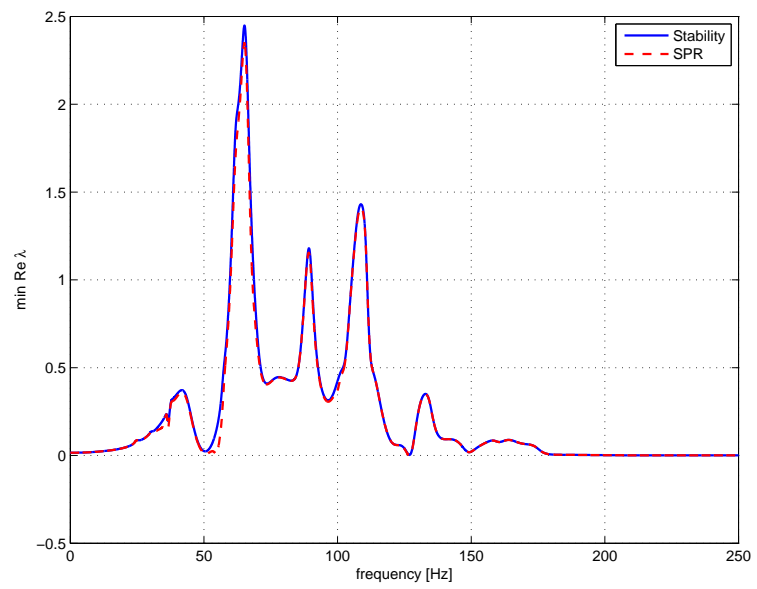

Figure 12: Minimum real eigenvalue of Eqs. (3) and (5) in which $G$ is the original secondary path and in which $G$ is the secondary path with damping obtained by $L Q R$ state feedback.

state feedback is given in Figs. 12 - 16. In this case the weighting matrices $Q$ and $R$ for the LQR regulator were $Q=I$ and $R=10^{3} I$. These values ensure that all real parts of the eigenvalues are positive and also that the resulting curve of the real part of the eigenvalue vs. frequency has approximately the same smoothness as in the nominal situation. The LQR regulator was applied to the minimum-phase factor $G_{o}$, leading to a modified minimum-phase factor $G_{o}^{\prime}$. Subsequently, the modified transfer function $G^{\prime}$ was obtained by inclusion of the original all-pass factor $G_{i}$ from $G^{\prime}=G_{i} G_{o}^{\prime}$. It can be seen that, except for very low frequencies below $7 \mathrm{~Hz}$, the stability condition is satisfied, i.e. the minimum real part of the eigenvalues is positive. However, the SPR condition is not always fulfilled. Nevertheless, the minimum eigenvalues for the SPR condition have been made less negative due to the LQR feedback. For the very low frequencies for which still a negative real part of the eigenvalues exists, an alternative stabilization technique is required. Frequency dependent regularization is considered, which should ensure that the minimum real part of the eigenvalues becomes positive at the low frequencies while having a minimum influence at higher frequencies. The shape of the frequency dependent regularization is shown in Fig. 17. The corresponding transfer function was multiplied with a constant such that a high-frequency regularization level of $-10 \mathrm{~dB}$ was obtained. The resulting minimum real part of the eigenvalues can be found in Fig. 18. Because all values of the stability curve are positive the controller is stable. However, the SPR condition still results in negative values for some frequencies with possible overshoot of the error signal during convergence. 


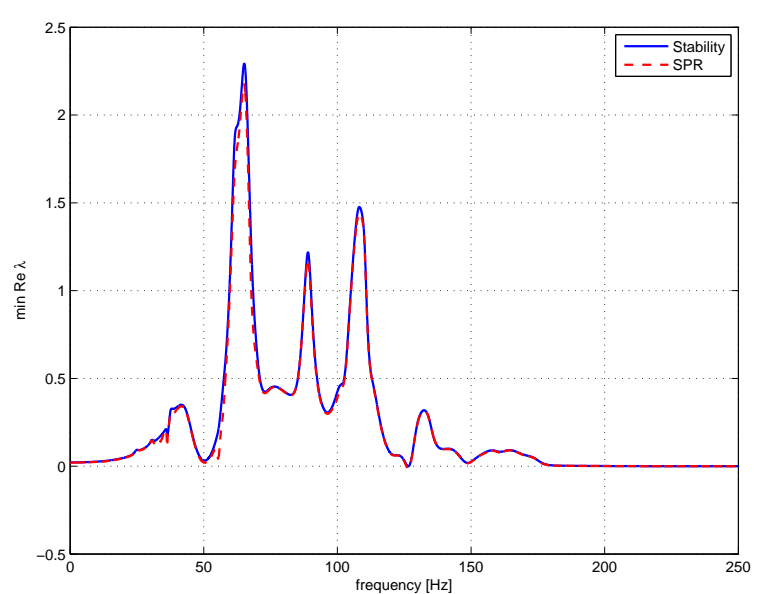

Figure 13: As Fig. 12, except that $G$ was obtained one hour later.

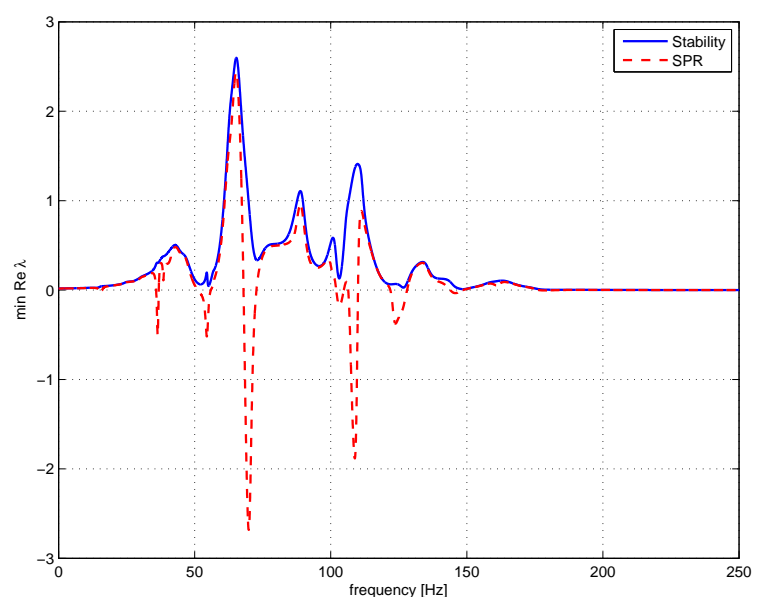

Figure 14: As Fig. 12, except that $G$ is obtained with the door open.

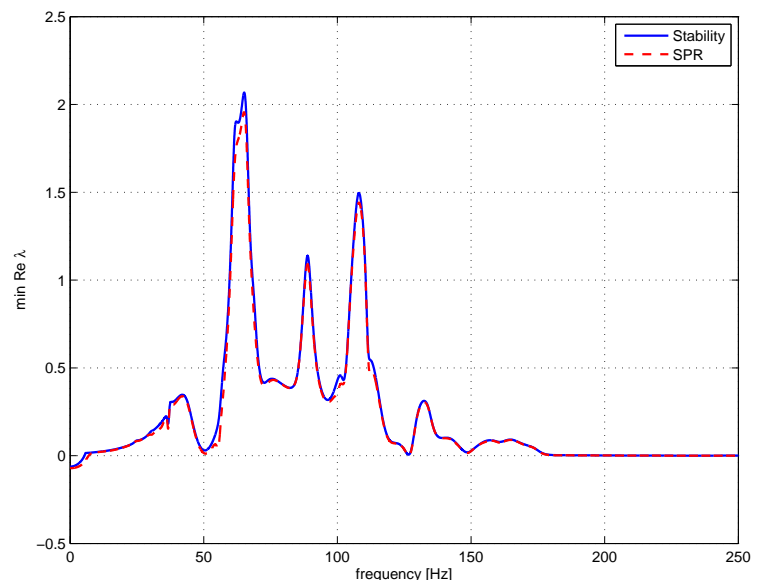

Figure 15: As Fig. 12, except that $G$ is obtained with the window open.

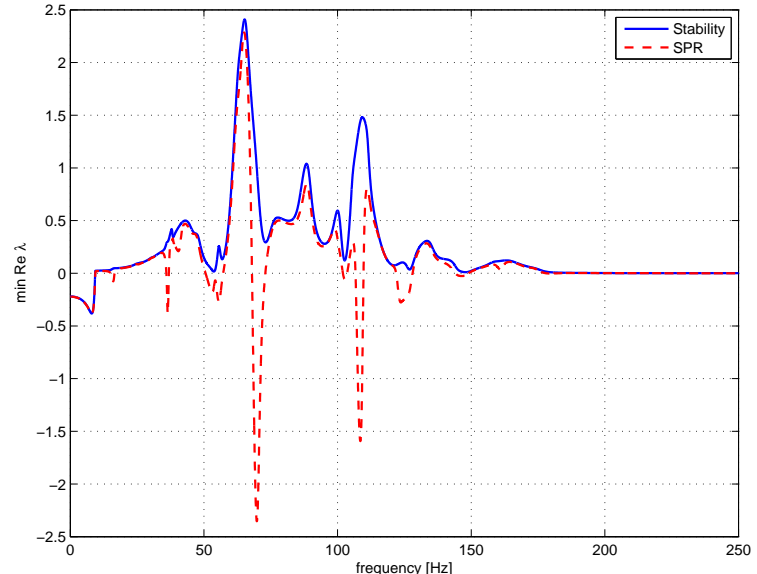

Figure 16: As Fig. 12, except that $G$ is obtained with the door and the window open.

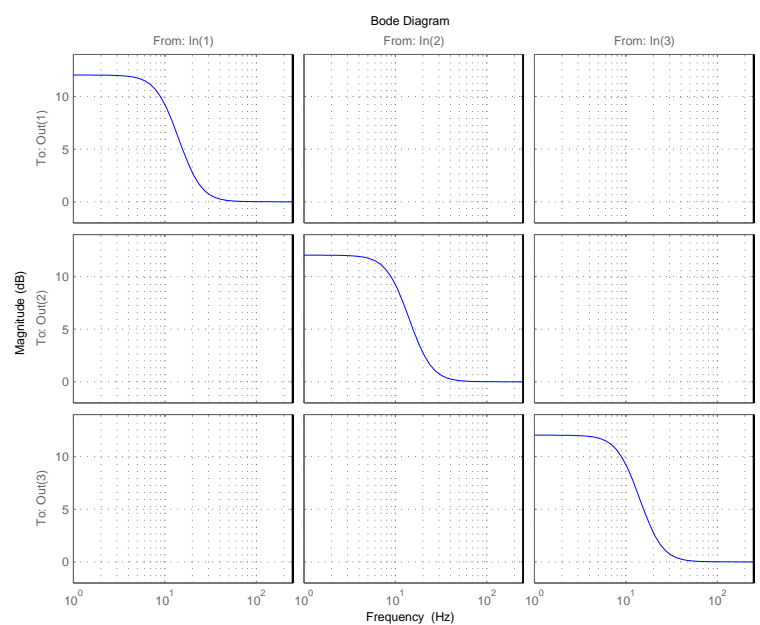

Figure 17: Magnitude of $G_{\mathrm{reg}}$ as used for frequency dependent regularization. In this case, the high-frequency regularization level equals $0 \mathrm{~dB}$.

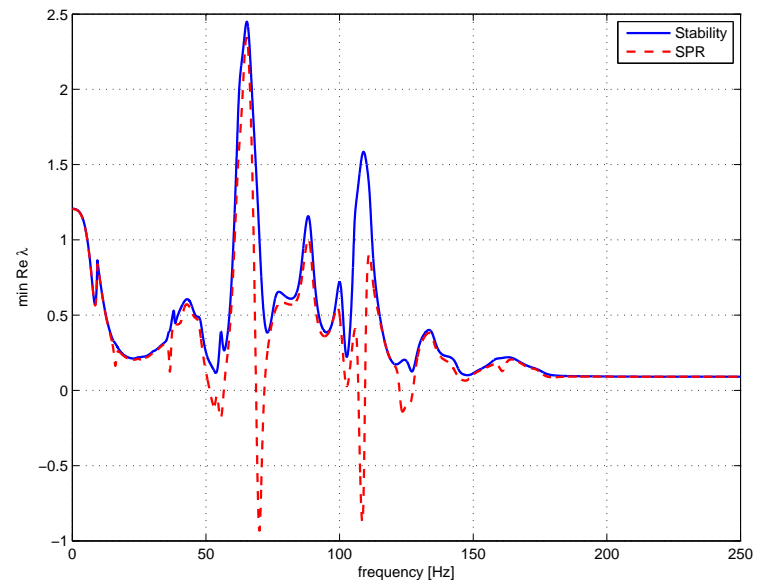

Figure 18: As Fig. 16, using the frequency dependent regularization shape of Fig. 17 multiplied by a constant such that the high-frequency regularization level becomes $-10 \mathrm{~dB}$. 


\section{Simulation results}

Using the measured transfer functions as described in the previous section, simulations were performed to verify the robustness as predicted by the stability analysis for different control strategies. Furthermore, the final reduction of the error signals was determinded for a converged algorithm in a stationary situation. The results are shown in Table 1 . The nominal condition denotes the situation in which all windows and doors are closed. The modified condition denotes the situation in which the windows and doors are opened. The nominal controller denotes a controller which uses the model obtained during the nominal condition and which uses effort weighting, i.e. frequency independent weighting. In the case that damping is used, damping is applied to the nominal model. Frequency dependent weighting is also used in combination with the nominal model, with or without damping. Frequency dependent weighting is based on frequency independent weighting for frequencies above $20 \mathrm{~Hz}$ with additional amplification for frequencies below $10 \mathrm{~Hz}$ using a 2nd-order filter, as shown in Fig. 17. This regularization technique emphasizes regularization at lowfrequencies while being less conservative at higher frequencies. At low frequencies the gain of the frequency dependent regularization filter is $12 \mathrm{~dB}$ higher than at high frequencies. The regularization level for frequency dependent weighting as indicated in the table is the value at high frequencies. The primary field was obtained by providing three independent white noise signals with a delay of 20 samples to the inputs of the nominal model or the modified model, depending on the condition that was used. The reference signals were the signals from the noise generators. The algorithm of Ref. [11] was used with affine projection order $K_{A}=4$, delay length $N_{d}=150$, number of controller coefficients for each channel $W_{i}=250$, leakage coefficient $\gamma=10^{-5}$, affine projection regularization parameter $\delta=0.25$, convergence coefficient $\alpha=0.025$. A convergence coefficient higher than 0.025 led to somewhat faster convergence in case there was no model mismatch. However, such a higher convergence coefficient resulted in less robustness and higher error signals in case of model mismatch. The convergence of the new algorithm is shown in Fig. 19, whereas the convergence for the old algorithm for the same situation is shown in Fig. 20.

In Table 1, it can be seen that adding damping to the secondary path model has a positive effect on the reduction of the error signals that can be achieved. Damping also has a positive effect on the stability of the system in the sense that a lower value of regularization level is possible for stabilizing the system. For the modified condition, the highest reductions of the error signals are possible when a combination is used of added damping and frequency dependent regulariza-

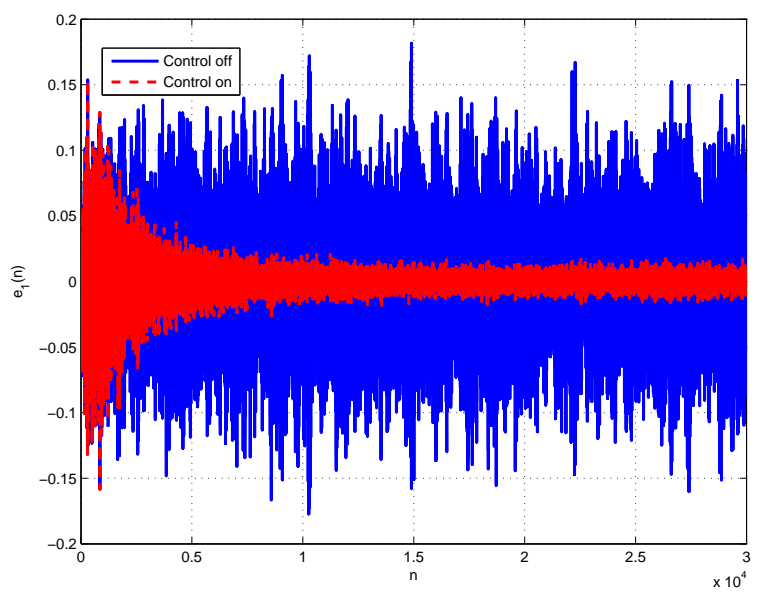

Figure 19: First error signal $e_{1}(n)$ with control and without control (i.e., $e_{1}(n)=d_{1}(n)$ ) for the situation with a model obtained in the nominal situation and a controller operated in the modified situation with added damping and frequency dependent regularization showing stable behavior.

tion, leading to maximum MSE reductions of $21.6 \mathrm{~dB}$ to $26.4 \mathrm{~dB}$ (marginally stable). For the nominal controller the maximum reduction for the same condition is $12.0 \mathrm{~dB}$, i.e. considerably less. Frequency dependent regularization alone does not improve the noise reduction for this case. The addition of damping leads to an improvement, in this case $16.8 \mathrm{~dB}$ maximum reduction. Subsequent addition of frequency dependent regularization leads to a further possible improvement of the reduction of the error signals. These results are in agreement with the stability analysis of the previous section. Also the regularization levels that are needed for stabilization are in agreement with the results of the previous section. Remarkable is that damping also has a positive effect on the amount of reduction in the nominal situation. Additional simulations were performed with longer filter lengths, i.e. higher values of $N_{d}$ for the realization of the delayed adjoint operator $D G^{*}$. However, this did not result in higher noise reductions. A possible explanation could be that errors in the modeling of undamped poles is critical and that, in order to avoid computed gradients with large errors, it is advantageous to use cautious gradients based on poles which are assumed to have more damping.

For the present configuration, a high-frequency regularization level of $-10 \mathrm{~dB}$ yields good performance for the nominal situation $(21.7 \mathrm{~dB}$ reduction) as well as for the situation with model mismatch $(21.6 \mathrm{~dB}$ reduction). Even when the model of the secondary path equals the real secondary path, the new scheme outperforms the nominal controller, which yields $20.7 \mathrm{~dB}$ reduction for the same regularization level at high frequencies. 
Table 1: Mean-square reduction of the error signals in dB after $1000 \mathrm{~s}$ for different physical situations (Condition), controller models and control strategies, and high-frequency regularization level. A dash indicates an unstable system, an asterisk indicates marginal stability.

\begin{tabular}{|c|c||c|c|c|c|c|c|c|c|}
\hline \multirow{2}{*}{ Condition } & \multicolumn{1}{c||}{ Model, control strategy } & \multicolumn{9}{c|}{ Regularization level [dB] } \\
\cline { 3 - 10 } & nominal & 9.7 & 13.4 & 17.3 & 20.7 & 22.5 & 23.1 & 23.2 & 23.2 \\
\hline \hline nominal & freq.dep.reg. & 9.5 & 12.9 & 16.5 & 19.6 & 21.7 & 22.7 & 23.2 & 23.2 \\
\hline nominal & damping & 9.5 & 13.6 & 18.4 & 23.9 & 29.4 & 33.5 & 36.4 & 36.8 \\
\hline nominal & nominal & 8.6 & 12.0 & - & - & - & - & - & - \\
\hline nominal & damping, freq.dep.reg. & 9.3 & 13.2 & 17.3 & 21.7 & 26.3 & 30.7 & 35.9 & 36.8 \\
\hline modified & freq.dep.reg. & 8.5 & 11.8 & - & - & - & - & - & - \\
\hline modified & damping & 8.5 & 12.2 & 16.8 & - & - & - & - & - \\
\hline modified & damping, freq.dep.reg & 8.4 & 12.0 & 16.4 & 21.6 & $26.4^{*}$ & - & - & - \\
\hline modified & & & & & & & -10 & - & - \\
\hline
\end{tabular}

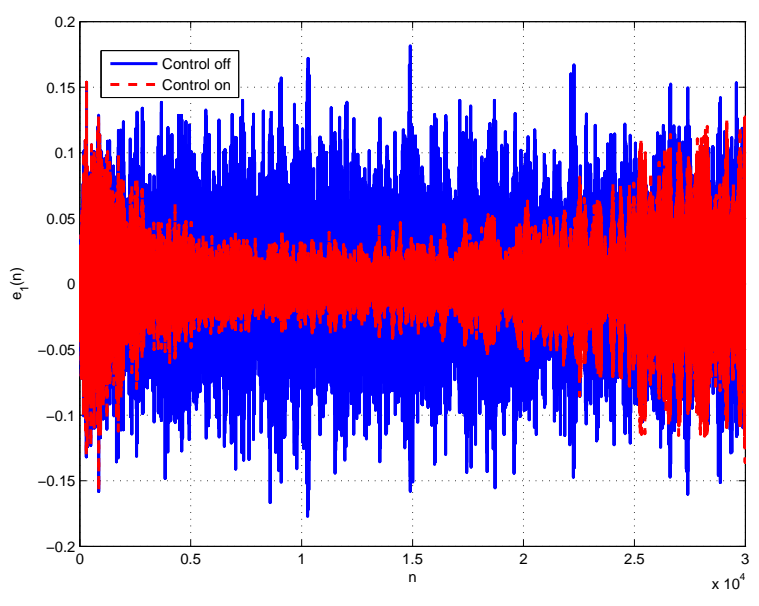

Figure 20: First error signal $e_{1}(n)$ with control and without control (i.e., $\left.e_{1}(n)=d_{1}(n)\right)$ for the situation with a model obtained in the nominal situation and a controller operated in the modified situation without added damping and without frequency dependent regularization showing unstable behavior.

\section{Concluding remarks}

In this paper the performance of an adaptive feedforward controller was investigated in which the controller was modified with frequency dependent regularization and in which transfer function models with increased damping were used. It was found that the combined controller modifications of adding damping and a frequency dependent regularization lead to improved performance as compared to adding damping only or regularization only. The scheme improves the stability for the case that the secondary path model differs from the real secondary path. Furthermore, the technique leads to higher possible reductions of the error signal. For the configuration considered in this paper good performance is obtained for the nominal situation as well as for the situation with model mismatch. Even when the model of the secondary path is identical to the real secondary path, the new scheme outperforms the nominal controller. A practical advantage of the scheme is that it does not require detailed uncertainty models using additional system identification cycles for each individual installation of the system.

\section{References}

[1] S.J.Elliott. Active Sound and Vibration Control, chapter 3, Adaptive methods in active control, pages 57-72. IET, London, 2002.

[2] K. Zhou, J. C. Doyle, and K. Glover. Robust and Optimal Control. Prentice Hall, Upper Saddle River, New Jersey 07458, 1996.

[3] M. Sternad and A. Ahlen. Robust filtering and feedforward control based on probabilistic descriptions of model errors. Automatica, 29:661679, 1993.

[4] B. Bernhardsson. Robust performance optimization of open loop type problems using models from standard identification. Systems and Control Letters, 25:79-87, 1995.

[5] B. Rafaely and S. J. Elliott. A computationally efficient frequency-domain $1 \mathrm{~ms}$ algorithm with constraints on the adaptive filter. IEEE Transactions on Signal Processing, 48:1649-1655, 2000.

[6] S. J. Elliott. Signal processing for active control. Academic Press, 2001.

[7] A. Preumont, A. Francois, F. Bossens, and A. Abu-Hanieh. Force feedback versus acceleration feedback in active vibration isolation. $J$ Sound Vib, 257:605-613, 2002.

[8] S. Herold, D. Mayer, and H. Hanselka. Transient simulation of adaptive structures. Journal of Intelligent Material Systems and Structures, 15:215-224, 2004.

[9] E. A. Wan. Adjoint lms: an efficient alternative to the filtered-x lms and multiple error lms algorithms. In Proc. Int. Conf. on Acoustics, Speech and Signal Processing ICASSP96, pages 18421845, Atlanta, 1996. IEEE. 
[10] A. P. Berkhoff and J. M. Wesselink. Rapidly converging adaptive state-space-based multichannel active noise control algorithm for reduction of broadband noise. J Acoust Soc Am, 121:3179, 2007.

[11] J. M. Wesselink and A. P. Berkhoff. Fast affine projections and the regularized modified filterederror algorithm in multichannel active noise control. J Acoust Soc Am, 124:949-960, 2008.

[12] M. Morari and E. Zafiriou. Robust Process Control. Prentice-Hall, London, 1989.

[13] W. Ren and P. R. Kumar. Adaptive active noise control: Structures, algorithms and convergence analysis. In Proc. Internoise 89, pages 435-440, Newport Beach, CA, 1989. INCE.

[14] P. R. Fraanje, S. J. Elliott, and M. Verhaegen. Robustness of the filtered-x lms algorithm - part ii: Robustness enhancement by minimal regularization for norm bounded uncertainty. IEEE Transactions on Signal Processing, 55:4038-4047, 2007.

[15] A. P. Berkhoff and J. M. Wesselink. Combined mimo adaptive and decentralized controllers for broadband active noise and vibration control. Mechanical Systems and Signal Processing, 25:1702-1714, 2011.

[16] A. Preumont. Vibration control of active structures. Kluwer Academic Publishers, Dordrecht, 1997.

[17] P. V. Overschee and B. D. Moor. Subspace identification for linear systems. Kluwer Academic Publishers, 1996. 